\title{
Parameterized Mobile Action Generator For A Wireless PCS Network
}

\author{
Sang-Eon Park and Carla Purdy \\ Department of Electrical and Computer Engineering and Computer Science \\ University of Cincinnati \\ Email: spark@ececs.uc.edu, carla.purdy@uc.edu
}

\begin{abstract}
While there are different research groups in the mobile computing community, most research requires mobile action data in terms of user calling and mobility patterns. Since collection of such real data is currently difficult and since most activity data is proprietary [12], researchers need to model this mobile activity data to evaluate their work. In [9] we model parameterized mobile actions in a wireless personal communication service (PCS) network based on three components: human behavioral daily movements cycle (traffic and labor), calling patterns, and topological localities. We have developed a mobile action generator based on the model. Our goal in designing the mobile action generator is to provide a common "benchmark" through which researchers can directly exchange performance results and avoid inaccuracies. We describe our model and the structure of our mobile action generator and present simulation results showing the performance of several call setup protocols.
\end{abstract}

\section{Background}

The PCS network generally requires pre-configured infrastructures (Figure 1) such as base stations (BS) and mobile switch centers (MSC). Each whole service area for an MSC is divided into several smaller service regions, hexagonal cells. In each cell, at least one base station is allocated to provide network service to mobile hosts in the cell. The mobile subscriber connects to the network by establishing a wireless connection to the base station. The connections among base stations are usually provided by a high speed wired backbone. The end-to-end delivery of a data packet relies mostly on the technology available for the wired backbone. The MSC is the central authority and does most processing. In particular it is responsible for coordinating the broadcast of system parameters, processing registrations, querying the location registers, managing paging, selecting the nearest base station, establishing connections, coordinating handoff between base stations and MSC's and so on.

Each subscriber is assigned a permanent home zone. At any given time, the user location information is stored in a database in the user's home zone called the Home Location Register (HLR), and also in a database in his current zone called the Visitor Location Register (VLR). Our model is based on this infrastructure [4].

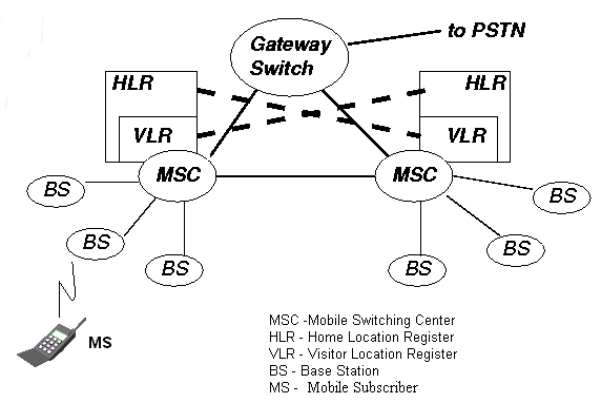

Figure 1. Wireless PCS network infrastructure

\section{Modeling Parameterized Mobile Actions}

\subsection{Topological Model}

A topological model provides a measurable location structure for mobile management scheme simulation. We define the topological area as a two dimensional space. An MSC zone is a zone covered by an MSC. Each MSC zone is defined by a rectangle which could be represented with two points in the two dimensional space. The average area $\left(\right.$ mile $\left.^{2}\right)$ covered by an MSC zone is used as a parameter for defining a distance unit for macroscopic movement (mobility model, see Section 2.5). Any geographical area covered by a wireless PCS infrastructure could be merged into our topological model. 


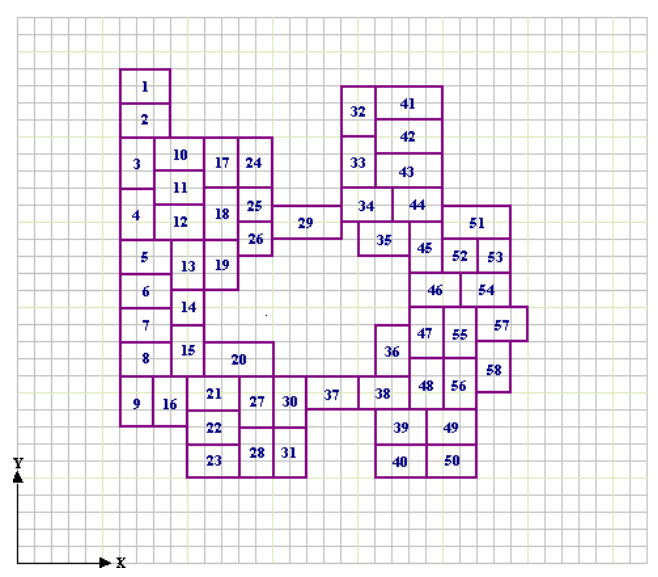

Figure 2. Imaginary geographical areas merged into topological model

Figure 2 shows an imaginary geographical area merged into our topological model. There are $58 \mathrm{MSC}$ zones. Two points $\mathrm{P}_{\mathrm{i}}\left(\mathrm{x}_{\mathrm{Pi}}, \mathrm{y}_{\mathrm{Pi}}\right)$ and $\mathrm{Q}_{\mathrm{i}}\left(\mathrm{x}_{\mathrm{Qi}}, \mathrm{y}_{\mathrm{Qi}}\right)$ represent a zone covered by $\mathrm{MSC}_{\mathrm{i}}$. Each subscriber is registered in one MSC zone for services.

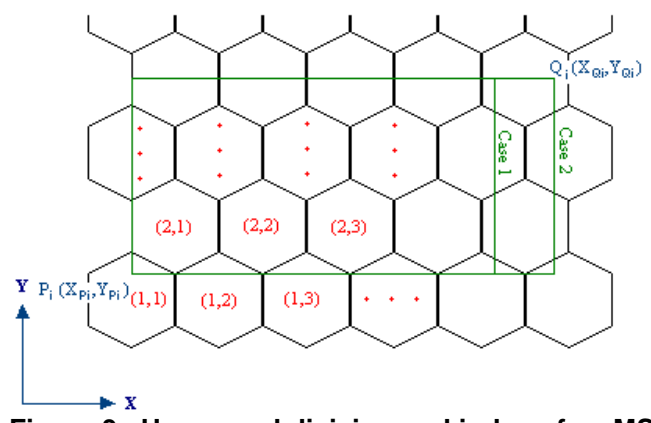

Figure 3. Hexagonal division and index of an MSC zone

Each MSC zone is divided into hexagonal cells and indexed for location management (Figure 3). In the center of each cell, one base station is allocated to provide network service to mobile hosts in the cell. Hexagonal cell size is also a parameter for indexing cells in each MSC zone.

\subsection{Subscriber Model}

The location of a person at a certain time could be estimated with his/her daily life style. Most people usually have two term periods: rest term period and working term period. People are usually staying home or roaming around near the home area during the rest term period. On the other hand, they move to the working area or work during the working term period. Their regular movement characteristics are considered for modeling mobile actions.

We classify subscribers into two groups: predictable and unpredictable. A predictable subscriber is a subscriber who has a regular stationary job; his/her location could be estimated during his/her working term period. On the other hand, an unpredictable subscriber is a subscriber who has an irregular job or regular but mobile job; his/her location could not possibly be estimated during his/her working term period. The proportion of predictable and unpredictable subscribers is used as a parameter for creating a macroscopic timetable of each subscriber.

\subsection{Macroscopic Timetable}

The macroscopic timetable of each subscriber describes his/her macroscopic daily schedule. It contains type of subscriber (predictable or unpredictable), rest zone, working zone (in the case of predictable subscriber), working period, and rest period of a subscriber. These entries could be estimated from possible traffic and labor distribution data $[5,6,7]$.

\subsubsection{Distance Distribution Between Work Zone and Rest Zone}

Any geographical area, merged into our topological model, could be represented by a graph as follows. We say that two MSC zones are adjacent if they share an edge (more than a single point) in our topological model.

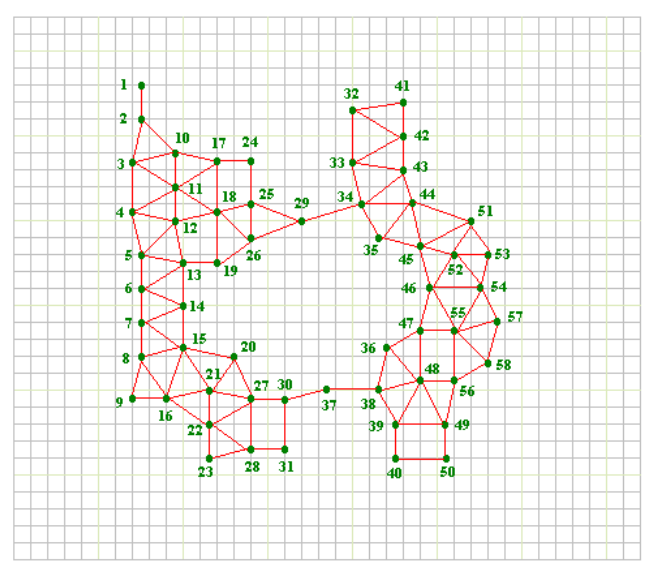

Figure 4. Corresponding graph for Figure 2

Figure 2 shows a topological area covered by 58 MSC's. Figure 4 is its corresponding graph $\boldsymbol{G}=(\boldsymbol{V}, \boldsymbol{E})$ where $\boldsymbol{V}=\left\{v_{1}, v_{2}, v_{3}, v_{4}, \ldots, v_{58}\right\}$. If $\mathrm{v}_{\mathrm{i}}$ and $\mathrm{v}_{\mathrm{j}}$ are 
adjacent MSC's, G has an edge $\left(\mathrm{v}_{\mathrm{i}}, \mathrm{v}_{\mathrm{j}}\right)$ of weight 1 . The number of distance units between $\mathrm{MSC}_{\mathrm{i}}$ and $\mathrm{MSC}_{\mathrm{j}}$ is the sum of weights on the shortest path between the two zones in G. For example, from Figure 2, the number of distance units between $\mathrm{MSC}_{2}$ and $\mathrm{MSC}_{34}$ is 6 since a shortest path from $\mathrm{MSC}_{2}$ to $\mathrm{MSC}_{34}$ is $\left(\mathrm{v}_{2} \Rightarrow\right.$ $\left.\mathrm{v}_{3} \Rightarrow \mathrm{v}_{11} \Rightarrow \mathrm{v}_{18} \Rightarrow \mathrm{v}_{25} \Rightarrow \mathrm{v}_{29} \Rightarrow \mathrm{v}_{34}\right)$ from Figure 4 . The shortest path between two MSC zones is also used for macroscopic movement (see Section 2.5) between two MSC zones.

From the travel time to work distribution [5], the distance unit distribution between rest zone and working zone can be estimated. Let $\boldsymbol{A}_{\boldsymbol{A v g}}\left(\right.$ mile $\left.^{2}\right), \boldsymbol{S}_{\boldsymbol{M a c}}$ (mile/minute), $\boldsymbol{L}_{\text {avg }}$ (miles) and $\boldsymbol{D}_{\boldsymbol{H a v g}}$ (minute) denote an average area covered by an MSC, average macroscopic movement driving speed, the average distance for a subscriber crossing a zones, and the average driving hours for a subscriber to change service zones respectively. To simplify, $\boldsymbol{L}_{\boldsymbol{a v g}}$ is defined as $\sqrt{A_{\text {Avg }}}$ (miles). With $\boldsymbol{S}_{\boldsymbol{M a c}}$ and $\boldsymbol{L}_{\boldsymbol{A v g}}, \boldsymbol{D}_{\boldsymbol{H} \boldsymbol{A v g}}$ could be defined as $D_{\text {HAvg }}=\frac{L_{\text {Avg }}}{S_{\text {Mac }}}(\min u t e)$

From the travel time to work distribution and $\boldsymbol{D}_{\boldsymbol{H A v g}}$, we can get an estimated distance unit distribution between rest and work zone.

From $D_{H A v g}$ and the travel time to work distribution of $U . S$ [5], the distance unit distribution between rest zone and working zone could be estimated (Table 1 with $\boldsymbol{A}_{\text {Avg }}=300\left(\mathrm{mile}^{2}\right), \boldsymbol{S}_{\text {Mac }}=50 \mathrm{mile} /$ hour $)$.

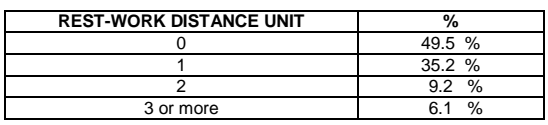

Table 1. Distance unit distribution between rest zone and working zone

The distance unit distribution between rest and work zone is a parameter for setting each subscriber's rest and working zone in macroscopic timetable.

\subsubsection{Work Starting Time Distribution}

Different people work at different times. Working starting time distribution is used as a parameter for setting each subscriber's working period in the macroscopic timetable. Work starting time distribution could be estimated from distribution of time leaving home to go to work [6].

According to the travel time to work distribution of U.S [5], 93.9\% of people take less than 60 minutes to get to work and average travel time to work is 22.4 minutes. From this a distribution of work starting time can be calculated. Table 2 shows work starting time distribution estimated from the distribution of time leaving home to go to work [6] and the travel time to work distribution of U.S [5].

\begin{tabular}{|c|c|c|}
\hline Work Starting Time & Number & Percent (\%) \\
\hline Workers 16 years and over ........ & $115,070,274$ & \\
\hline Did not work at home .................. & $111,664,249$ & 100.0 \\
\hline 6:00 a.m. to $6: 59$ a.m $\ldots \ldots \ldots . .$. & $7,145,946$ & 6.4 \\
\hline 7:00 a.m. to $7: 59$ a.m ........... & $22,820,464$ & 20.5 \\
\hline $8: 00$ a.m. to $8: 59$ a.m .......... & $35,346,620$ & 31.7 \\
\hline 9:00 a.m. to $9: 59$ a.m .......... & $18,867,326$ & 16.9 \\
\hline $10: 00$ a.m. to $10: 59$ a.m ........ & $5,792,355$ & 5.2 \\
\hline $11: 00$ a.m. to $11: 59$ a.m ............. & $2,249,960$ & 2.0 \\
\hline $12: 00$ a.m. to $12: 59$ a.m ...... & $1,167,633$ & 1.0 \\
\hline $1: 00$ p.m. to $4: 59$ p.m ........... & $7,965,160$ & 7.1 \\
\hline 5:00 p.m. to $12: 59$ p.m ......... & $7,561,297$ & 6.8 \\
\hline 1:00 a.m to $5: 59$ a.m ............... & $2,747,488$ & 2.5 \\
\hline Worked at home & $3,406,025$ & \\
\hline
\end{tabular}

Table 2. Work starting time distribution estimated by time leaving home to go to work

\subsubsection{Working Hour Distribution}

Table 3 shows a daily working hour distribution estimated from the person at work, by hours worked distribution [7] (we assume that people work 5 days a week). The daily working hour distribution is also a parameter for setting each subscriber's working period in the macroscopic timetable.

\begin{tabular}{|c|c|c|}
\hline $\begin{array}{l}\text { Hours of Work } \\
\text { (daily) }\end{array}$ & $\begin{array}{c}\text { Person at work } \\
(1,000)\end{array}$ & $\begin{array}{c}\text { Percent } \\
\text { distribution }\end{array}$ \\
\hline $\bar{T}$ Total $\ldots \ldots \ldots \ldots \ldots \ldots \ldots$ & 125,877 & 100.0 \\
\hline 0 to 3 Hours ........... & 6,250 & 5.0 \\
\hline 3 to 6 Hours ........... & 15,545 & 12.3 \\
\hline 6 to 7 Hours ........... & 11,337 & 9.0 \\
\hline 7 to 8 Hours ........... & 8,731 & 6.9 \\
\hline 8 Hours ................ & 44,224 & 35.1 \\
\hline 8 to 9 Hours ........... & 14,608 & 11.6 \\
\hline 9 to 10 Hours ........ & 14,520 & 11.5 \\
\hline over 10 Hours........ & 10,663 & 8.5 \\
\hline
\end{tabular}

Table 3. A daily working hour distribution

Now macroscopic timetable entries could be estimated from the distribution data discussed previously. Rest zone of each subscriber is randomly chosen. Work zone for a predictable subscriber could be selected based on rest-work zone distance unit distribution. Working period and rest period could be estimated by rest-work zone distance unit distribution, work starting time distribution and working hour distribution.

\subsection{Call Model}

The call model generates call traffic for each subscriber. It is characterized by two parts: the call traffic distribution and the callee distribution. The call traffic distribution is a proportion of busy subscribers and available subscribers at a time period of a day. The call traffic is used as a parameter for generating mobile action data. In Table 4 we show example traffic distribution data obtained from [10]. 


\begin{tabular}{|c|c|}
\hline Time Period & Average Busy Rate \\
\hline $12 \mathrm{AM} \sim 04 \mathrm{AM}$ & $05 \%$ \\
\hline $04 \mathrm{AM} \sim 08 \mathrm{AM}$ & $10 \%$ \\
\hline $08 \mathrm{AM} \sim 12 \mathrm{PM}$ & $25 \%$ \\
\hline $12 \mathrm{PM} \sim 04 \mathrm{PM}$ & $30 \%$ \\
\hline $04 \mathrm{PM} \sim 08 \mathrm{PM}$ & $20 \%$ \\
\hline $08 \mathrm{PM} \sim 12 \mathrm{AM}$ & $08 \%$ \\
\hline
\end{tabular}

Table 4. Call traffic distributions

The callee distribution describes how a subscriber chooses a callee. Most callers usually do not choose a callee randomly; they usually have their frequent calling number list. In our model, each subscriber is combined with a frequent calling number list. The probability that a subscriber calls someone on his/her frequent calling number list is also a parameter in our call model for choosing a callee in our model. We also consider "mutual favorites" to construct the frequent calling number list of each subscriber.

\subsection{Mobility Model}

There are several mobility models designed for human movement. In the fluid model $[8,11]$, traffic flow is shown as the flow of a fluid. The Markovian model [1] describes individual subscriber movements. The gravity model [2] has been used to model human movement behavior in transportation research. These models are proper in some areas, but not for wireless PCS mobility. There is no concept of trips or consecutive movements through a series of regions in any of these models. Trip is an important modeling aspect when considering the mobility model.

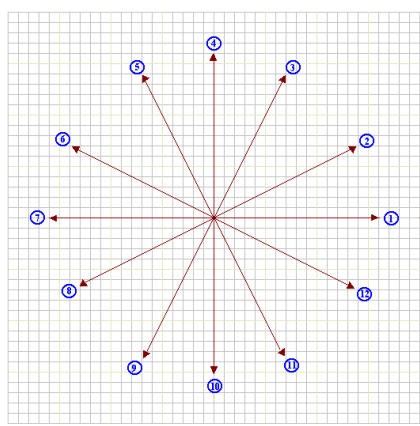

a) Movement directions

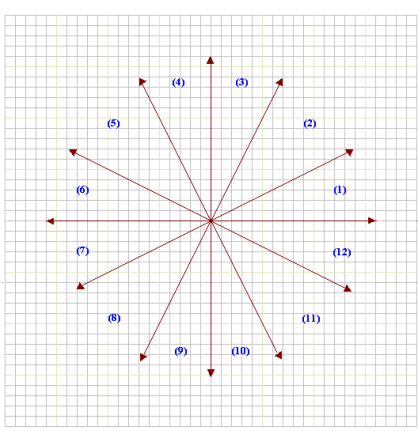

b) Destination locations
Figure 5. Twelve movement and destination directions

Our mobility model uses goal-oriented movement. Every human's movement usually has goals to achieve. Once he/she sets a goal, he/she starts to move to achieve the goal. Once the goal is achieved, he/she might set a new goal and start to move, and so on. Each movement goal depends on movement type. We define two movement types: macroscopic and microscopic movement. Each subscriber's movement type is determined by current time, his/her timetable and subscriber type (predictable or unpredictable). Twelve movement and destination directions (Figure 5) are possible for the next movement.

\subsubsection{Microscopic Movement}

Microscopic movement, which is combined with a destination direction and duration, is usually a movement inside an MSC zone. The movement goal is to go forward to the destination direction during its duration. During the movement duration, next movement direction is chosen based on the destination direction.

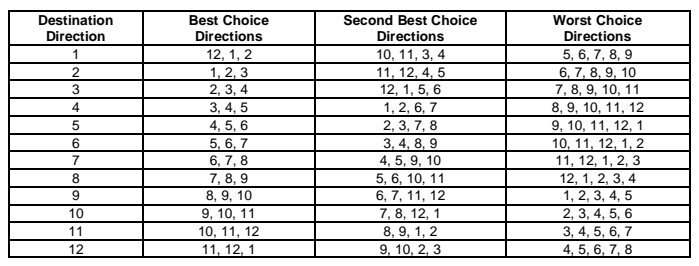

Table 5. Microscopic movement directions

Table 5 is built according to human movement tendency with possible roads. We say that a direction is a possible direction if the next location caused by the movement to that direction with average microscopic (or macroscopic) movement speed is still in any valid service zone. We start with best choice directions (from Table 5) based on microscopic direction. If there is more than one possible direction, one will be chosen randomly for next movement direction. Else, choose from the second best choice direction, and so on. When the duration is expired, a goal is archived and a new movement type will be chosen based on current time, his/her timetable and subscriber type.

\subsubsection{Macroscopic Movement}

Macroscopic movement is a movement that causes MSC service zone changes. The goal is to reach a destination zone. Suppose a subscriber's current location is $M C_{10}$ (MSC zone 10) and he/she wants to go to location $\mathrm{MSC}_{35}$ (Figure 2). If he/she goes from $\mathrm{MSC}_{10}$ to $\mathrm{MSC}_{35}$ in a random way, it could take forever. Therefore, we define rules for macroscopic movement. Macroscopic movement between two zones follows a shortest path (Figure 4). So the macroscopic 
movement path from $\mathrm{MSC}_{10}$ to $\mathrm{MSC}_{35}$ is $\mathrm{MSC}_{10}$ $\begin{array}{llllll}\mathrm{MSC}_{11} & \mathrm{MSC}_{18} & \mathrm{MSC}_{25} & \mathrm{MSC}_{29} & \mathrm{MSC}_{34} & \mathrm{MSC}_{35} .\end{array}$ Travel between adjacent zones in a macroscopic movement path is modeled by a sequence of steps. Each step is along a directional vector whose angle is an integral multiple of 30 degrees (Figure 5(a)). The direction of the next intermediate step is chosen as follows. The set of twelve possible directions is divided into three sets of size four based on how well they agree with the angle of the vector which goes directly from the current location to the center point of the next service zone (Figure 5(b)). If any of the four vectors from the best choice set places the subscriber in any valid service zone, then one of those valid vectors is chosen randomly. Otherwise a choice is made from the valid vectors in the next best set and so on (Table 6). This step is repeated until the subscriber reaches the adjacent zone.

\begin{tabular}{|c|c|c|c|}
\hline $\begin{array}{c}\text { Destination } \\
\text { Zone Location } \\
\text { (center of a zone) }\end{array}$ & $\begin{array}{c}\text { Best Choice } \\
\text { Directions }\end{array}$ & $\begin{array}{c}\text { Second Best } \\
\text { Choice } \\
\text { Directions }\end{array}$ & $\begin{array}{c}\text { Worst Choice } \\
\text { Directions }\end{array}$ \\
\hline 1 & $12,1,2,3$ & $10,11,4,5$ & $6,7,8,9$ \\
\hline 2 & $1,2,3,4$ & $11,12,5,6$ & $7,8,9,10$ \\
\hline 3 & $2,3,4,5$ & $12,1,6,7$ & $8,9,10,11$ \\
\hline 4 & $3,4,5,6$ & $1,2,7,8$ & $9,10,11,12$ \\
\hline 5 & $4,5,6,7$ & $2,3,8,9$ & $10,11,12,1$ \\
\hline 6 & $5,6,7,8$ & $3,4,9,10$ & $11,12,1,2$ \\
\hline 7 & $6,7,8,9$ & $4,5,10,11$ & $12,1,2,3$ \\
\hline 8 & $7,8,9,10$ & $5,6,11,12$ & $1,2,3,4$ \\
\hline 9 & $8,9,10,11$ & $6,7,12,1$ & $2,3,4,5$ \\
\hline 10 & $9,10,11,12$ & $7,8,1,2$ & $3,4,5,6$ \\
\hline 11 & $10,11,12,1$ & $8,9,2,3$ & $4,5,6,7$ \\
\hline 12 & $11,12,1,2$ & $9,10,3,4$ & $5,6,7,8$ \\
\hline
\end{tabular}

Table 6. Macroscopic movement directions

\section{Summary of Parameters}

Topological, subscriber, call and mobility models have been discussed in previous sections. Summarized parameters for generating our mobile action model follow:

Topological model parameters

a. Total number of MSC zones in geographical area

b. Average area of an MSC zone

c. Location of each MSC zone - an $\mathrm{MSC}_{\mathrm{i}}$ zone is represented by two points $\mathrm{P}_{\mathrm{i}}\left(\mathrm{x}_{\mathrm{p}}, \mathrm{y}_{\mathrm{pi}}\right)$ and $\mathrm{Q}_{\mathrm{i}}\left(\mathrm{x}_{\mathrm{q}}\right.$, $\left.\mathrm{y}_{\mathrm{qi}}\right)$

d. Number of subscribers registered in each MSC zone

e. Size of hexagonal cell

Subscriber model parameters

a. Work starting time distribution b. Proportion of predictable and unpredictable subscribers

c. Distribution of daily working hours

d. The distance unit distribution between rest zone and working zone

\section{Call model parameters}

a. Call traffic distribution

b. Call duration distribution

c. Mutual favorite rate

d. Traceable rate - proportion of traceable subscribers

\section{Mobility model parameters}

a. Movement speed - average walking and vehicle speed

b. Probabilities for choosing movement types based on a state

\section{Mobile Action Generator for a Wireless PCS Network}

We have developed a mobile action generator based on the model defined in [9]. It is written in ANSI C with approximately 4500 lines in Microsoft Visual Studio. It is combined with a topological description data file (parameter.dat), call distribution data files (BusyRate_Distri.dat, call_duration.dat), traffic distribution data files and behavioral distribution data files. Each subscriber's mobile action is composed of movement and calling action. The movement actions for each subscriber at any time are decided by the macroscopic timetable, current time and rules defined in the previous sections. The calling actions are based on the call volume distribution at the given time and the calling behavioral characteristics (calling list of each subscriber).

\subsection{Structure of Mobile Action Generator}

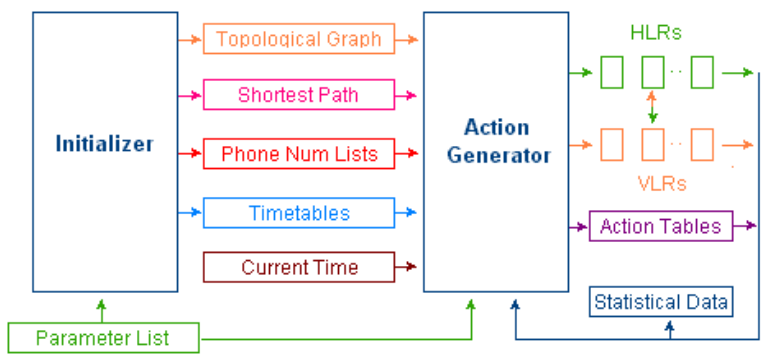

Figure 6. Mobile action generator 
Figure 6 shows the structure of our mobile action generator. The mobile action generator is composed of "Initializer" and "Action Generator". "Initializer" creates the topological graph based on the topological parameter list and calculates shortest paths from one vertex to every vertex that is used for the macroscopic movement. It also creates a favorite phone number list with a mutual favorite list and a timetable for each subscriber based on behavioral parameters and topological parameters. The "Action Generator" generates HLR/VLR scheme based on each subscriber's location and status (busy, avail). It also creates the action table that describes each subscriber's previous action and next action. In each cycle, the "Action Generator" updates the subscriber's next action according to current time, action table, current location, their timetable, favorite phone number list and behavioral parameters.

\subsection{Initializer}

The "Initializer" creates a topological graph according to topological locations of each MSC zone. The MSC's topological information is stored in the data file "parameter.dat". This graph is used for macroscopic movement between two zones based on shortest paths. There are several algorithms to solve the shortest path problem. We use Dijkstra's shortest path algorithm which solves the shortest-path problem on the positive weighted graph created.

A favorite phone number of a subscriber is a frequently called phone number. The "Initialzer" creates a favorite phone number list for each subscriber randomly. But it also considers the mutual favorite rate. If Bob is on Jim's favorite phone number list, then the probability that Jim's number is on Bob's frequent calling number list is high. We call this "mutual favorite rate".

The timetable of each subscriber is the daily macroscopic time schedule. It contains working period, resting period, rest zone and working zone (if predictable subscriber). It is used for each subscriber's macroscopic movement decisions at any time. The "Initializer" creates a timetable according to the distance unit distribution between rest zone and working zone (stored in the file "rw_dis_distri.dat"), the work starting time distribution (stored in the file wk_st_distri.dat) and the daily working hour distribution (stored in the file wk_hr_distri.dat).

\subsection{Action Generator}

The "Action Generator" creates the initial HLR/VLR scheme according to current time, current busy rate, timetable, and parameters (topological, behavioral). It also creates an action table for each subscriber. The action table for an each subscriber is created based on current time, the subscriber's timetable and the subscriber's status (busy, avail, untraceable) from HLR/VLR. Contents of the action table are current and next movement information (movement type, movement direction, movement duration (microscopic movement case), destination zone (macroscopic movement case) and calling information (calling duration). In each cycle, the "Action Generator" updates HLR/VLR according to the action table's next action information and predicts next actions based on current time, current status, timetable and movement parameters.

\section{Simulation}

One of the most important and challenging problems for wireless communication and computing is mobility management. Mobility management enables telecommunication networks to locate roaming terminals for call delivery and to maintain connections as the terminal is moving into a new service area. Thus, mobility management supports mobile terminals, allowing users to roam while simultaneously offering them incoming calls and supporting calls-in-progress.

Mobility management contains two components: location management and handoff management. There are two processes in location management that enable the network to discover the current attachment point of the mobile user for call delivery. With a call setup protocol, the network is queried for the user location profile and the current position of the mobile host is found. Location registration (or location update) is another process for location management. In location registration, the mobile subscriber periodically notifies the network of its new access point, allowing the network to authenticate the user and revise the subscriber's location profile.

There have been several call setup protocols proposed, we have simulated several call setup protocols with mobile actions created by our action generator.

\subsection{Call Setup Protocols}

Several steps are taken during a call setup, and we define each step as a separate procedure. For each procedure, the number of database lookups, wired network messages, and network hops are counted to measure call setup performance. When a database is queried for user location information, it counts as one database lookup. Every remote database lookup, 
connection setup request, or acknowledgment contributes one network message. An inter-zone message may take multiple network hops to reach its destination. There have been several call setup protocols proposed.

\section{IS-41}

In the IS-41 [2] standard, when a caller calls a callee, the caller initiates a local lookup at the local VLR for the callee's information. If the callee's information is not found, the local VLR sends a query to the callee's home HLR where the callee's VLR address is always stored. Then the callee's HLR retrieves the callee's routing and status information from the callee's current VLR and sends it back to the local VLR where the caller is currently located. If the callee is not busy, the connection request is sent from the caller's MSC to the callee's MSC, and the network path is reserved. The callee is then paged in his cell. The call setup is completed once the caller receives a response from the callee; otherwise the call setup fails.

\section{Lightweight Location Lookup Protocol (LiLLP)}

In Lightweight Location Lookup Protocol (LiLLP) [3], the location lookup stage in the call setup from a caller to a callee retrieves only the callee's VLR address, instead of his entire routing information, thereby reducing the tasks of the location lookup stage. After obtaining the callee's VLR address, the system sends a connection setup request to the callee's VLR and reserves network paths. Upon receiving the connection setup request, the callee's VLR forwards the request to the callee's MSC, according to the routing information stored in the VLR. The callee's MSC, which is colocated with the VLR in most current systems, pages the callee in his cell. The connection setup is completed once the callee responds.

\section{In the Reverse Connection Setup (ReCS)}

In the Reverse Connection Setup (ReCS) [3] protocol, when a call is made from a caller to a callee, the system first finds the callee's current VLR address in the caller's local database or the callee's HLR, and sends the location lookup request to the callee's current VLR. The callee's current VLR then looks at its database for the callee's routing information and directly pages the callee instead of sending the routing information back to the caller as in IS-41. The circuit connection setup is initiated from the callee to the caller automatically (which is the reverse of the call request direction) after the callee answers.

\subsection{Simulation}

We use the following parameters. Topological model is from Figure 2 (58 MSC zones with $400\left(\right.$ mile $\left.^{2}\right)$ average area). Total number of subscribers is 16,100. (The number of subscribers our model can handle is limited only by memory size, as is the number of MSC zones). Work starting time distribution, distribution of daily working hours, the distance unit distribution between rest zones and working zone are from Table 1, Table 2 and Table 3 respectively. Proportions of predictable and unpredictable subscribers are $30 \%$ predictable subscribers and $70 \%$ unpredictable subscribers. Call traffic distribution is from Table 4. This simulation took about 24 hours on a $400 \mathrm{MHz}$ Pentium II. Table 7 and Figure 7 show the simulation results of the three call setup protocols with mobile actions created by our mobile action generator over a 24 hour period.

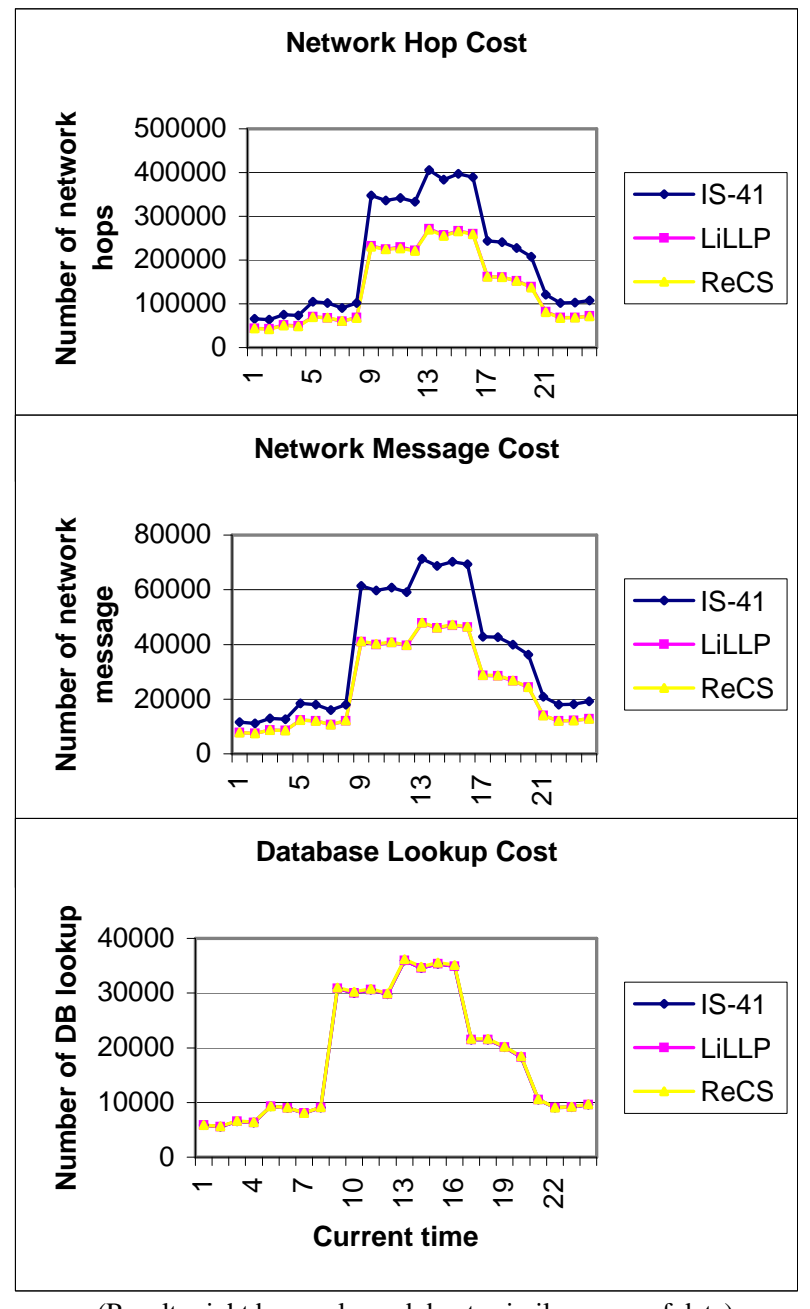

(Result might be overlapped due to similar range of data) Figure 7. Simulation results for call setup protocols 


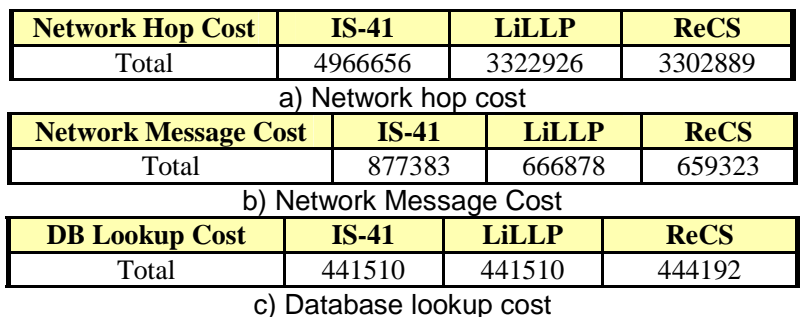

Table 7. Simulation result for call setup protocols

Network hop costs in our simulation are about $10-15 \%$ less than those found analytically in [3]. This is probably due to the fact that, in our model, geographically adjacent zones are always considered to have a network connection. This simplifying assumption could be replaced by the more complex assumption that a certain percentage of geographically adjacent areas will not have a direct network connection. Our simulation results for network message cost and database cost are similar with the results of [3]. According to the simulation [3], LiLLP improves about $27 \%$ overall and ReCS improves about $29 \%$ overall of network message cost. And there is no difference in database lookup cost. In our simulation, LiLLP improves about $24 \%$ and ReCS improves about $25 \%$ in network message cost. And there is also no difference in database lookup cost.

\section{Conclusions and Future Work}

We have presented a realistic framework for modeling mobile actions in a wireless PCS network and a mobile action generator based on our framework. The framework incorporates human behavioral distribution data to relate the performance of our model to real human actions. Simulation results showing the performance of several call setup protocols are presented. We are currently using our mobile action generator to study mobility management algorithms for wireless PCS networks.

\section{References}

[1] A. Bar-Noy and I. Kessler. Mobile users: To update or not to update? Proceedings of INFOCOM'94 Conference on Computer Communications, Vol. 2, pp. 570-576, June 1994.

[2] C. Ben-Noy, R. Bouchard and E. S. Jr. Clyde. An evaluation of simplified procedures for determining travel patterns in a small urban area. Computer
Simulation of Human Behavior, chapter 20, pages 400-430. John Wiley\&Sons, Inc., 1971.

[3] Y. Cui, D. Lam, J. Widom, and D.C. Cox. Efficient PCS Call Setup Protocols. In Proceedings of the 1998 IEEE Conference on Computer Communications (Infocom '98), vol3, pages 728736, San Francisco, California, March 1998S.

[4] EIA/TIA, Cellular radio-telecommunications intersystem operations, Technical Report IS-41 Revision C, EIA/TIA, 1995

[5] 1990 Census of Population, STF3C. The travel time to work distribution of U.S. in 1990 Journey-toWork and Migration Statistics Branch, Population Division, U.S. Bureau of the Census.

[6] 1990 Census of Population, STF3C. The distribution of time leaving home to go to work in 1990 Journey-to-Work and Migration Statistics Branch, Population Division, U.S. Bureau of the Census.

[7] Bureau of Labor Statistics, the person at work, by hours worked distribution Employment and Earnings, monthly, January, 1999 issue.

[8] K.K. Leung, W.A. Massey and W. Whitt. Traffic models for wireless communication networks. IEEE Journal on Selected Areas in Communications, 12(8): 1353-1364, October 1994.

[9] Sang-Eon Park and C. Purdy, Modeling Parameterized Mobile Actions in Wireless Personal Communication Services Networks, Proceedings of the IEEE International Conference on Third Generation Wireless and Beyond (IEEE 3Gwireless'2001), San Francisco May 30 - June 2, 2001, pp $60 \sim 66$.

[10] Telephone call traffic data set,11/94-7/95 S. Phillips, personal communication, Oct 1995.

[11] Victor S. Frost and B. Melamed. Traffic modeling for telecommunications network. IEEE Communication Magazine March 1994, pp.70 - 81.

[12] PLEIADES Project www-db.stanford.edu/ jan/HiDB.html 